\title{
Cluster Based Optimization of Routing in Distributed Sensor Networks Using Bayesian Networks with Tabu Search
}

\author{
Lokesh B. Bhajantri and Nalini N.
}

\begin{abstract}
Distributed Sensor Networks (DSNs) have attracted significant attention over the past few years. A growing list of many applications can employ DSNs for increased effectiveness especially in hostile and remote areas. In all applications a large number of sensors are expected and requiring careful architecture and management of the network. Grouping nodes into clusters has been the most popular approach for support scalability in DSN. This paper proposes a cluster based optimization of routing in DSN by employing a Bayesian network (BN) with Tabu search (TS) approach. BN based approach is used to select efficient cluster heads and construction of $\mathrm{BN}$ for the proposed scheme. This approach incorporates energy level of each node, bandwidth and link efficiency.

The optimization of routing is considered as a design issue in DSN due to lack of energy consumption, delay and maximum time required for data transmission between source nodes (cluster heads) to sink node. In this work optimization of routing takes place through cluster head nodes by using TS. Simulations have been conducted to compare the performance of the proposed approach with LEACH protocol. The objective of the proposed work is to improve the performance of network in terms of energy consumption, throughput, packet delivery ratio, and time efficiency of optimization of routing. The result shows that the proposed approach perform better than LEACH protocol that utilizes minimum energy, latency for cluster formation and reduce overhead of the protocol.
\end{abstract}

Keywords-Distributed Sensor Networks (DSN), Routing, Cluster Head (CH), Bayesian Network (BN) and Tabu Search (TS)

\section{INTRODUCTION}

A DSN is a collection of a large number of heterogeneous intelligent sensors distributed logically, spatially or geographical over an environment and connected by a high speed network. Recent advancement in micro-electronics and telecommunication technologies has been resulted in design of tiny sensing nodes that are capable of continuously collect data from their respective environments. The collected data are processed by an associated processing element that transmits it through an interconnected communication network. Sensors are deployed varies with the applications $[1$, 2]. The sensor are deeply embedded devices that are integrated with a physical environment and capable of acquiring signal, processing the signals, communicating, routing of data and

Lokesh B. Bhajantri is with the Department of Information Science and Engineering, Basaveshwar Engineering College, Bagalkot, Karnataka, India (e-mail: lokeshcse@yahoo.co.in).

Nalini N. is with the Department of Computer Science and Engineering, Nitte Meenakashi Institute of Technology, Bangalore, India (e-mail: nalinaniranjan@hotmail.com). performing computation tasks. While this new class of networks has the potential to enable wide range of applications and poses serious challenges like frequent network topology change, limited computational, memory and optimization of routing.

A randomly deployed sensor network requires a cluster formation protocol to partition the network into clusters to increase the network lifetime and optimization of energy level of sensor nodes. In clustering nodes are grouped into clusters in the network. Each cluster has at least one $\mathrm{CH}$ with instead of sending data directly to base station (BS). Nodes send data to their corresponding $\mathrm{CH}$ via single or multi hop communication. $\mathrm{CH}$ receives data of all its nodes in each cluster for transmission to sink node. Clustering of nodes avoid long distance communication of nodes to BS. We have considered as an optimization of routing in DSN in the proposed scheme. The optimization of routing is an important parameter in critical applications like military, environment monitoring, disaster management, etc., where routing of data is significant. Some of routing challenges and design issues in DSN as follows: network dynamics, node deployment, energy considerations, data delivery models, node capabilities, data aggregation or fusion. Many routing protocols are considered in DSN environment as follows: Sensor Protocols for Information via Negotiation (SPIN), Low-Energy Adaptive Clustering Hierarchy (LEACH), Geographic and Energy Aware Routing (GEAR), and Greedy Perimeter Stateless Routing (GPSR) [3, 4, 5, 6, 7].

In this work we have proposed $\mathrm{BN}$ approach is used for $\mathrm{CH}$ selection and construction of BNs. The proposed model is used to represent conditional independences between a set of random variables ( $\mathrm{CHs}$ or clusters). $\mathrm{BN}$ consists of a set of variables and directed edges between variables, each variable has finite set of mutually exclusive states and variables together with the edges forms a directed acyclic graph (DAG). Apart from this, TS approach based routing protocol is used to optimize the routing in the DSN. One of the main components of TS is its use of adaptive memory, which creates a more flexible search behavior and it improves the ability to locate optimal solutions. It is an iterative search that starts from some initial feasible solution and attempts to determine the best solution. The TS algorithm keeps historical local optima for leading to the near global optimum fast and efficiently and it choose a feasible solution at random and then obtain a neighbor to this solution. During these search procedures the 
best solution is always updated and stored aside until the stopping criterion is satisfied. The TS has two prominent features are: adaptive memory and responsive exploration strategies. In this algorithm, always move to the best available neighborhood solution point, even if it is worse than the current solution point. The aim is to minimize the computational time and improvement of quality of the solution in the communication $[8,9]$.

The rest of the paper is organized as follows: Section 2 presents an overview of related work. A proposed work for system architecture, cluster formation and $\mathrm{CH}$ selection, construction of network and optimization routing is discussed in Section 3. Simulation and results analysis are presented in Section 4 and finally we conclude the proposed work in Section 5.

\section{RELATED WORK}

Some of the related works are as follows: The work given in [10] presents a novel cluster based routing protocol in wireless sensor networks. To construct a spanning tree for sending aggregated data to the base station by using network parameters. Simulation results shows that CBRP can remarkably extend the network lifetime and amount of data gathered. Energy efficient fire monitoring over cluster based wireless sensor networks is presented in [11]. It proposes EFMP (Energy efficient Fire monitoring Protocol), a fire monitoring protocol operating over cluster based sensor networks. The work given in [12] depicts a new clustering protocol for wireless sensor networks using Genetic Algorithm approach. This work examines the optimization of the lifetime and energy consumption of wireless sensor Networks (WSNs).

The work given in [13] discusses on distance based energy efficient clustering for wireless sensor networks. This work addresses the new distributed clustering and data aggregation algorithm. A simulation result shows that the proposed algorithm balances the energy dissipation over the whole network thus prolongs the network lifetime. Clustering method for energy efficient routing in wireless sensor networks is presented in [14]. It avoids creating redundant cluster heads within a small geographical range. Simulation result shows that proposed scheme reduces energy dissipation and prolongs network lifetime as compared with LEACH. The work given in [15] presents an energy-aware, cluster-based routing algorithm (ECRA) for wireless sensor networks. The ECRA selects some nodes as cluster-heads to construct voronoi diagrams and rotates the cluster-head to balance the load in each cluster. A two-tier architecture (ECRA-2T) is also proposed to enhance the performance of the ECRA.

The work given in [16] presents a Bayesian decision model for intelligent routing in sensor networks. The proposed solution is used to design a new efficient energy-aware routing algorithm based on learning patterns for sensor network that minimizes the main constraints imposed by this kind of networks. The probabilistic decision model both considered the estimation of the available energy at the neighboring nodes and the importance of the messages to make intelligent decisions. The work given in [17] depicts a distributed
Bayesian algorithm for fault-tolerant event region detection in wireless sensor networks. The proposed solution is used to detect the fault in the form of Bayesian fault-recognition algorithms. It presented two Bayesian algorithms, the randomized decision scheme and the threshold decision scheme, and derived analytical expressions for their performance. Bayesian fusion algorithm for inferring trust in wireless sensor networks is presented in [18]. This work introduces a new Bayesian fusion algorithm to combine more than one trust component (data trust and communication trust) to infer the overall trust between nodes. The proposed algorithm is simple and generic as it allows trust components to be added and deleted easily. Simulation results demonstrate that a node is highly trustworthy provided that both trust components simultaneously.

The work given in [19] presents a TS algorithm for cluster building in wireless sensor networks. It proposes a new centralized clustering method for a data collection mechanism in wireless sensor networks, which is based on network energy maps and quality-of-service (QoS) requirements. Simulation result shows that TS-based approach returns high-quality solutions in terms of cluster cost and execution time. Ant colony optimization routing algorithm with Tabu search is given [20]. It proposes a new hybrid routing algorithm which combines Tabu search with ant colony optimization. The proposed hybrid technique enables to find the shortest route in the network. Experiments prove the effectiveness in comparison with conventional routing algorithm such as Dijkstra algorithm. The work given in [21] discusses a TS WSN deployment method for monitoring geographically irregular distributed events. This paper addresses the wireless sensor network deployment issue. Integrating Genetic Algorithm, TS approach for job shop scheduling is given in [22]. It presents a new algorithm based on integrating Genetic Algorithms and TS methods to solve the job shop scheduling problem. The idea of the proposed algorithm is derived from Genetic Algorithms. Some of the related works are given in $[23,24]$.

A lot of study has been done in the area of energy efficient cluster based routing for WSNs using LEACH algorithm. Most of the earlier works based on the fault tolerance by using BNs. Very few works on the basis of BN to select $\mathrm{CH}$ in WSN but these works considers distance probabilistically between nodes, which are less efficient to select $\mathrm{CHs}$. The proposed approach incorporates energy level of each node, bandwidth and link efficiency. Earlier works do not consider the BN based cluster head selection with TS for optimization of routing in DSN environment. Several LEACH enhancements were proposed which appears to solve some of the shortcomings of LEACH by using different methodology and algorithms. The $\mathrm{CH}$ selection process in $\mathrm{LEACH}$ is random which a result is less optimal selection of $\mathrm{CHs}$ due to its stochastic nature, $\mathrm{CH}$ selection process is not optimal. However, it results in non - optimal utilization of resources. In this work, proposed $\mathrm{BN}$ inspired $\mathrm{CH}$ selection in DSN to increase the efficiency of cluster head selection and increase the network lifetime. Also proposed TS algorithm based 
inspired routing protocol (CBTS) for optimization of routing over the scheme, while discovering the optimization of routing from the source nodes to base node or sink node to increase the efficiency of the network. The following are the important assumptions made in the proposed scheme: (1) Nodes are stationary and deployed in a random fashion. (2) It is assumed that all nodes in the network are static and have initial energy. (3) All nodes are equipped with GPS. During set up phase all nodes convey its location and energy information to the BS. (4) Nodes sense the data periodically and send it to the sink node with optimization of routing using TS inspired routing protocol. (5) To simulate the concept of forced heads or forced cluster heads (FCHs) are acts as a $\mathrm{CH}$ in the network.

\section{PROPOSED WORK}

The proposed system replaces the existing system and to find efficient $\mathrm{CH}$ selection and construction of $\mathrm{BN}$ along with optimization of routing using TS approach over CHs. TS algorithm approach can be simulated more effectively. In this section we have discussed the system architecture, cluster formation and $\mathrm{CH}$ selection, functioning scheme, inferences or conditional probability tables and TS algorithm for finding optimization of routing in DSN.

\section{A. System Architecture}

The proposed system architecture consists of following components are: sensor nodes, $\mathrm{CHs,} \mathrm{forced} \mathrm{heads} \mathrm{or} \mathrm{forced}$ $\mathrm{CHs}$, and sink node or base station. The proposed system architecture consists of set of sensor nodes $\mathrm{S}=\left\{\mathrm{S}_{1}, \mathrm{~S}_{2}, \mathrm{~S}_{3}\right.$, -$\left.\mathrm{S}_{\mathrm{N}}\right\}$, which are placed randomly as shown in fig.1. Each node is assigned with the initial amount of the energy and the bandwidth. It is assumed that all nodes (sensor and sink nodes) in the network are static and sensor nodes are computing the path in the DSN. There are two types of communication in the proposed network such as: inter-cluster communication and intra cluster communication. Communication between the member nodes and their respective $\mathrm{CHs}$ are called as intra cluster communication, where as communication between the $\mathrm{CHs}$ to sink node is regarded as inter-cluster communication. Network is built by using the network configuration parameters. Each node sense the data periodically send it to the $\mathrm{CH}$ node, $\mathrm{CH}$ node transmits the data to the sink node with optimization of routing by using TS. The operation of the proposed scheme is as follows: (1) Create DSN environment randomly, (2) create the cluster by using Eq. (2), (3) to select the cluster head among nodes in the clusters with highest probability by using BN approach, (4) to construct the BN for proposed scheme with directed acyclic graph (DAG) i.e. no cycles in the graph due to avoid of delay for finding optimization of routing, (5) to find the optimization of routing using cluster based TS (CBTS) protocol in the network, and (6) Sink node takes the action upon receiving the information form sensor nodes.

This work considers the concept of FH or FCHs. Sensor nodes that do not come under of any chosen heads are by default considered as FCHs in the DSN. Also FH or FCHs are considered in the optimization of routing for data over the network. Hence the proposed scheme is used to solve a sensor network optimization problem and enhance the network lifetime over the scheme.

\section{B. Cluster Formation}

In which, nodes are deployed randomly in the area. Let ' 1 ' and ' $b$ ' be the length and breadth of the square area respectively. The area is divided into number of square area by using Eq.(1), to find the centre of the defined square area by using Eq.(2):

$$
\text { Centre of the square area }=(1 * b) / 2
$$

$$
\text { Cluster Formation }=\sum_{n=1}^{n} \mathrm{X}^{2}+\mathrm{Y}^{2}=\mathrm{R}^{2}
$$

where,

$$
\mathrm{R}=200 \mathrm{~m}
$$

$\mathrm{X}$ and $\mathrm{Y}$ are the coordinates of the centre of the clusters.

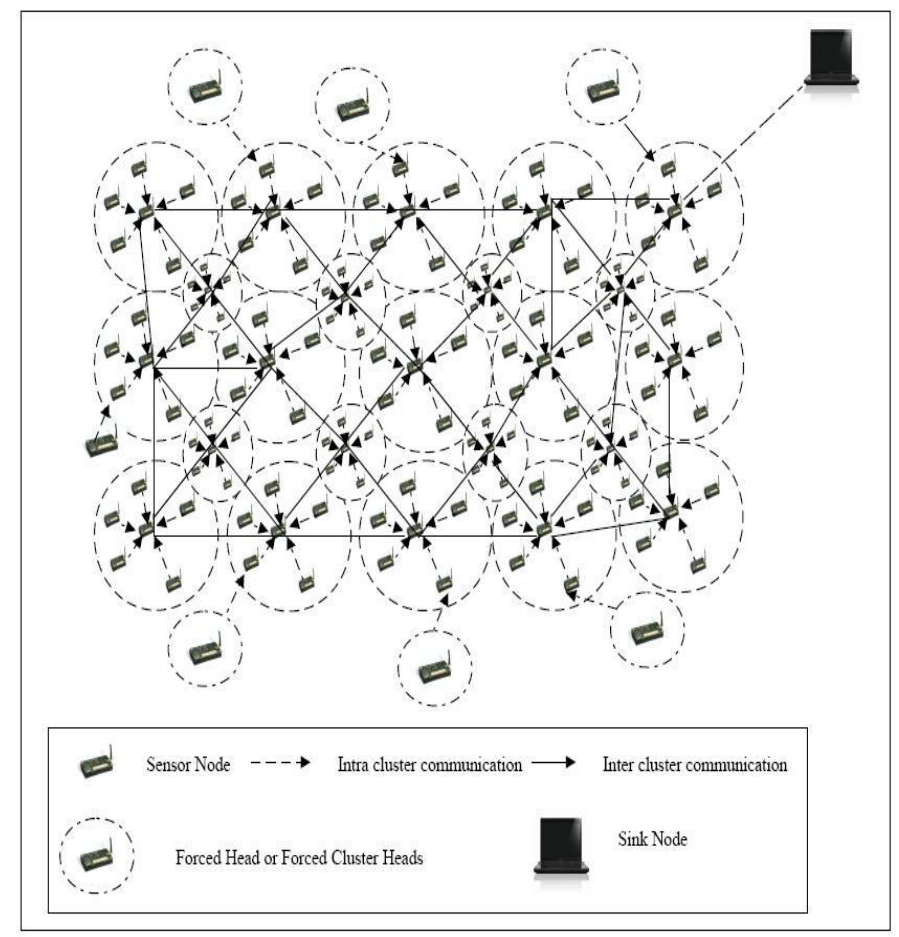

Fig.1. System Architecture

\section{Functioning scheme}

In this section, functions of $\mathrm{BN}$ and $\mathrm{TS}$ algorithms are explained. Some of the notations used in the proposed scheme are as follows: 
TABLE I

NOTATIONS

\begin{tabular}{|l|l|}
\hline \multicolumn{1}{|c|}{ Notations } & \multicolumn{1}{c|}{ Descriptions } \\
\hline $\mathrm{S}_{\mathrm{N}}=\left\{\mathrm{S}_{1}, \mathrm{~S}_{2}, \mathrm{~S}_{3},----\mathrm{S}_{\mathrm{N}}\right\}$ & Set of Sensor Nodes \\
\hline $\mathrm{E}_{\mathrm{N}}$ & Energy of Sensor Nodes \\
\hline $\mathrm{C}_{1}, \mathrm{C}_{2}, \mathrm{C}_{3}, \ldots \mathrm{C}_{\mathrm{N}}$ & Number of clusters \\
\hline $\mathrm{C}_{\mathrm{N}}$ & Costs Between each Nodes \\
\hline $\mathrm{T}_{\mathrm{R}}$ & Transmission Range \\
\hline $\mathrm{E}_{\mathrm{S}}$ & Energy Required for Sensing of Data \\
\hline $\mathrm{E}_{\mathrm{T}}$ & $\begin{array}{l}\text { Energy Required for Transmission of } \\
\text { Data }\end{array}$ \\
\hline $\mathrm{P}$ & Packet \\
\hline $\mathrm{T}_{\mathrm{H}} \mathrm{E}$ & Threshold Level Energy \\
\hline $\mathrm{Ns}$ & Number of Sink Nodes \\
\hline Length & l( in meters) \\
\hline Breadth & b (in meters) \\
\hline $\mathrm{R}_{\mathrm{N}}$ & Number of Rounds \\
\hline $\mathrm{T}_{\mathrm{H}} \mathrm{P}$ & Threshold Level probability \\
\hline $\mathrm{P}\left(\mathrm{S}_{\mathrm{N}}\right)$ & Probability of Sensor Node \\
\hline
\end{tabular}

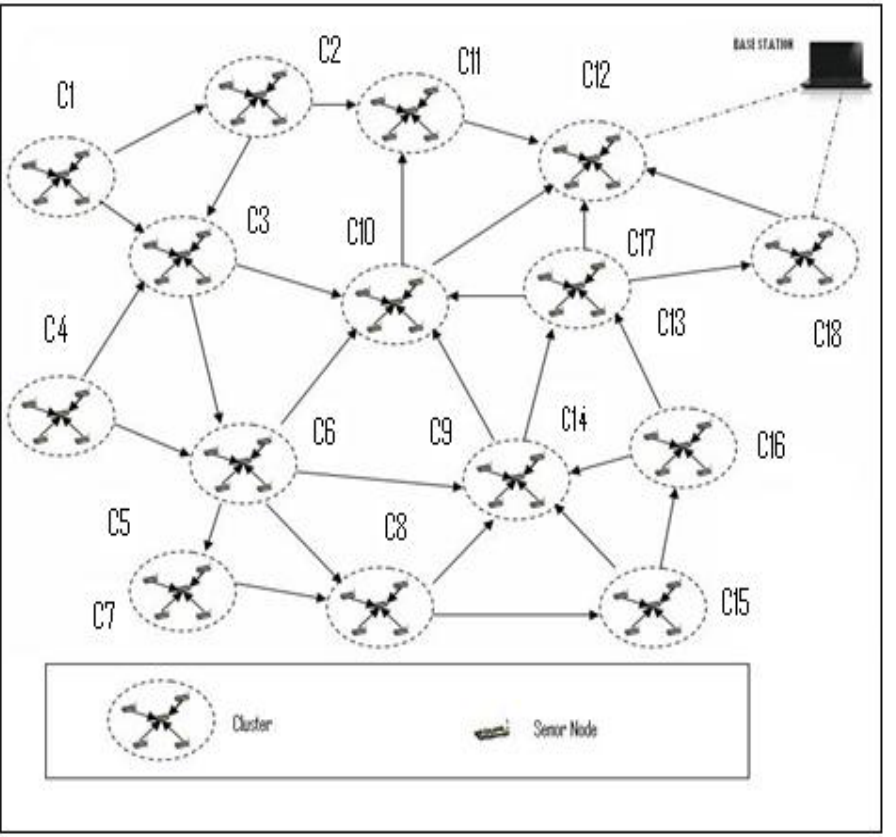

Fig.2. Bayesian Network for the Proposed Work

The above fig. 2 shows the $\mathrm{BN}$ for proposed scheme, in which the nodes (Clusters) represent the variables of $\mathrm{BN}$ and arcs indicates that probabilistic dependencies between nodes (Clusters). Also calculates the conditional probabilities for each nodes (Clusters). Root nodes (Clusters) do not have any parents and its uses the prior probability i.e. $P\left(C_{i}\right)$. The ordering guarantees that the $\mathrm{BN}$ will have no cycles. The procedure for construction of $\mathrm{BN}$ algorithm is given by:

\section{Procedure for Construction of Bayesian Network in proposed work}

1. Choose a set of variables (nodes) that describes the application domain
2. Choose an ordering of variables (Clusters or nodes) $\mathrm{C}_{1} \ldots \ldots \ldots \mathrm{C}_{\mathrm{N}}$

3. Start with the empty network and add variables (clusters) to the network in DSN environment

4. For $\mathrm{i}=1$ to $n$

Add $\mathrm{C}_{\mathrm{i}}$ to Network

Select parents from $\mathrm{C}_{1} \ldots \ldots \ldots \mathrm{C}_{\mathrm{N}}$ such as

$\mathrm{P}\left(\mathrm{C}_{\mathrm{i}} \mid\right.$ Parents $\left.\left(\mathrm{C}_{\mathrm{i}}\right)\right)=\mathrm{P}\left(\mathrm{C}_{\mathrm{i}} \mid \mathrm{C}_{\mathrm{i}} \ldots \ldots \ldots . . \mathrm{C}_{\mathrm{i}-1}\right)$

This choice of parent guarantees the global semantics:

$\mathrm{P}\left(\mathrm{C}_{1} \ldots \ldots \ldots \mathrm{C}_{\mathrm{N}}\right)=\Pi^{\mathrm{n}}{ }_{\mathrm{i}=1} \mathrm{P}\left(\mathrm{C}_{\mathrm{i}} \mid \mathrm{C}_{\mathrm{i}} \ldots \ldots \ldots \ldots \mathrm{C}_{\mathrm{i}-1}\right)$

//by chain rule

$$
=\mathrm{P}\left(\mathrm{C}_{1}\right) \mathrm{P}\left(\mathrm{C}_{2} \mid \mathrm{C}_{1}\right) \ldots \ldots . . \mathrm{P}\left(\mathrm{C}_{\mathrm{N}}\right.
$$

$\left(\mathrm{C}_{1}-----\mathrm{C}_{\mathrm{N}-1}\right)$

$$
=\Pi^{\mathrm{n}}{ }_{\mathrm{i}=1} \quad \mathrm{P}\left(\mathrm{C}_{\mathrm{i}} \mid \text { Parents }\left(\mathrm{C}_{\mathrm{i}}\right)\right)
$$

// by construction

5. Draw an arc from the each variables (clusters) in parents $\left(\mathrm{C}_{\mathrm{i}}\right)$ to $\mathrm{C}_{\mathrm{i}}$

This may be expressed as the product of the prior probabilities of all the root nodes (cluster) and the conditional probabilities of all the other nodes (clusters) in the network. The conditional probabilities are important for building BNs. But BNs are also built to facilitate the calculation of conditional probabilities, namely the conditional probabilities for variables (clusters) of interest given the data (evidence). Each variable (cluster) $C_{1}$ with parents $C_{2} \ldots . . C_{N}$ in a $B N$ has an attached conditional probability table $\mathrm{P}\left(\mathrm{C}_{1} \mid \mathrm{C}_{2} \ldots \ldots \mathrm{C}_{\mathrm{N}}\right)$, this table contains the probabilities for all possible combinations of the states of $\mathrm{C}_{1}$ and its parents. To reflect the interactions between the influences of multiple parents, a single conditional probability is assigned to each variable.

\section{E. Inferences or Conditional Probability Tables}

The given inferences are used in the process of deriving logical conclusion from premises known or assumed to be true. Here $\mathrm{BN}$ is used to determine the probabilities of particular types of events. Which is used to computing the conditional probability for variables with given information (evidence) concerning other nodes or variables. The evidence is available on ancestors of the variables or nodes of interests. The evidence is available on a descendant of the variable(s) or nodes (CHs) of interest to perform inference against the direction of the edges. The proposed Bayes' theorem is given by [25]:

$$
\mathrm{P}\left(\mathrm{S}_{2} \mid \mathrm{S}_{1}\right)=\left(\mathrm{P}\left(\mathrm{S}_{1} \mid \mathrm{S}_{2}\right) \mathrm{P}\left(\mathrm{S}_{2}\right)\right) / \mathrm{P}\left(\mathrm{S}_{1}\right)
$$

Let 'd'be the distance between nodes in the network. ' $\mathrm{d}_{\mathrm{i}}$ 'can be computed by using Euclidian Distance Formula (EDF) Eq. (4) $[26]$ :

$$
\mathrm{d}_{\mathrm{i}}=\sqrt{\left(x_{1}-x_{2}\right)^{2}+\left(y_{1}-y_{2}\right)^{2}}
$$


Link efficiency can be computed as follows. Let ' $\mathrm{C}_{\mathrm{i}}$ ' be capacity of a discrete-time discrete-valued channel, ' $\mathrm{B}$ ' be the bit rate $(\mathrm{Hz})$ of a channel (bandwidth), $\mathrm{E}_{\mathrm{T}}$ be the total energy consumed for transmission of a bit in link i, SNR be the signal-to-noise ratio [27]. Capacity of channel " $i$ ", is computed by using Eq. (5):

$$
\mathrm{C}_{\mathrm{i}}=\mathrm{Blog}_{2}(1+\mathrm{SNR})
$$

Let ' $\mathrm{E}_{\mathrm{N}}$ ' be the energy consumed for the transmission of a bit per distance ' $\mathrm{d}_{\mathrm{i}}$ '. Energy (E) can be computed by using Eq. (6) [27].

$$
\mathrm{E}=\mathrm{E}_{\mathrm{N}} * \mathrm{~d}_{\mathrm{i}}
$$

Let ' $\mathrm{L}_{\text {eff }}$ ' be the link efficiency for the nodes in the network. ' $\mathrm{L}_{\text {eff }}$ ' can be computed by using Eq. (7) [28].

$$
\mathrm{L}_{\mathrm{eff}}=\frac{C i}{E}
$$

Let ' $\mathrm{TE}_{\mathrm{N}}$ ' be the total energy consumption for the transmission of the packets. ' $\mathrm{TE}_{\mathrm{N}}$ ' can be computed by using Eq. (8)

$$
\mathrm{TE}_{\mathrm{N}}=\mathrm{S}_{\mathrm{E}} * \mathrm{P}_{\mathrm{i}} / \text { bits }+\mathrm{T}_{\mathrm{E}} * \mathrm{P}_{\mathrm{i}} / \text { bits }
$$

where,

$\mathrm{S}_{\mathrm{E}}=$ Energy required for sensing data or packets.

$\mathrm{T}_{\mathrm{E}}=$ Energy required for transmission of data or packets.

$\mathrm{P}_{\mathrm{i}}=$ Size of packets in terms of bits.

Let ' $\mathrm{R}_{\mathrm{E}}$ ' be the residual energy of each node. Let ' $\mathrm{R}_{\mathrm{E}}$ ' can be computed by using Eq. (9)

Where,

$$
\mathrm{R}_{\mathrm{E}}=\mathrm{I}_{\mathrm{E}^{-}} \mathrm{E}_{\mathrm{i}}
$$

$$
\begin{aligned}
& \mathrm{IE}=\text { initial energy of node } \\
& \mathrm{E}_{\mathrm{i}}=\text { Energy consumption }
\end{aligned}
$$

Total energy consumption of the path for optimization of routing over the $\mathrm{CHs}\left(\mathrm{E}_{\mathrm{CH}}\right)$. The total energy required by $\mathrm{CHs}$ to sink node over the path is given by

$$
\mathrm{E}_{\mathrm{CH}}=\mathrm{CH}_{\mathrm{i}} * 0.05 \mathrm{~nJ}
$$

Based on above equations are used to derive inferences for the proposed work as given below:

TABLE II

INFERENCES

\begin{tabular}{|c|c|c|c|}
\hline Energy & Distance & Bandwidth & Result \\
\hline Max & High & Min & High \\
\hline Max & Fair & Min & Fair \\
\hline Min & High & Min & Min \\
\hline Fair & High & Min & High \\
\hline Max & Min & Fair & Fair \\
\hline Max & Min & Max & Min \\
\hline
\end{tabular}

TABLE III

ENERGY VS CHANNEL CAPACITY

\begin{tabular}{|l|l|l|l|}
\hline & Low & Fair & High \\
\hline Min & 0.40 & 0.56 & 0.70 \\
\hline Fair & 0.55 & 0.74 & 0.85 \\
\hline Max & 0.60 & 0.78 & 0.97 \\
\hline
\end{tabular}

TABLE IV

BANDWIDTH VS DISTANCE

\begin{tabular}{|c|c|c|c|c|}
\hline & Low & Fair & High & Result \\
\hline Min & 0.55 & 0.80 & 0.96 & 2.31 \\
\hline Fair & 0.52 & 0.72 & 0.85 & 2.09 \\
\hline Max & 0.44 & 0.63 & 0.71 & 1.78 \\
\hline Result & 1.51 & 2.15 & 2.52 & \\
\hline
\end{tabular}

\section{F. Cluster Head Selection}

The probability of each sensor node become a $\mathrm{CH}$, which is based on its probabilistic from remaining nodes is computed. The one with the highest probability is selected as the $\mathrm{CH}$ in the network by using BN. The probability of each node is calculated based on residual energy, bandwidth and link efficiency. For finding the probability of each node by considering the following parameters: \{Energy, Bandwidth, and Link efficiency $\}$ in the proposed scheme.

Let ' $\mathrm{CH}_{\mathrm{i}}$ ' be the cluster head in the network. ' $\mathrm{CH}_{\mathrm{i}}$ ' can be selected by using Eq. (11). A conditional distribution for each node given it's by:

$\mathrm{CH}_{\mathrm{i}}=\mathrm{P}\left(\mathrm{S}_{1}, \mathrm{~S}_{2} \ldots \mathrm{S}_{\mathrm{N}}\right)=\prod^{\mathrm{n}}{ }_{\mathrm{i}=1} \mathrm{P}\left(\mathrm{S}_{\mathrm{i}} \mid\right.$ parents $\left.\left(\mathrm{S}_{\mathrm{i}}\right)\right)$

The conditional probability for each node is given by:

$\mathrm{P}\left(\mathrm{S}_{1} \mid \mathrm{S}_{2}, \mathrm{~S}_{3}, \mathrm{~S}_{\left.4-----\mathrm{S}_{\mathrm{N},}\right)}=\left(\mathrm{P}\left(\mathrm{S}_{1} \mid \mathrm{S}_{2}, \mathrm{~S}_{3}, \mathrm{~S}_{4---.-} \mathrm{S}_{\mathrm{N}}\right) * \mathrm{P}\right.\right.$

$\left.\left(\mathrm{S}_{2}, \mathrm{~S}_{3}, \mathrm{~S}_{4---.} \mathrm{S}_{\mathrm{N}}\right)\right) / \mathrm{P}\left(\mathrm{S}_{1}\right)$

\section{G. Cluster Head Selection for Each Round}

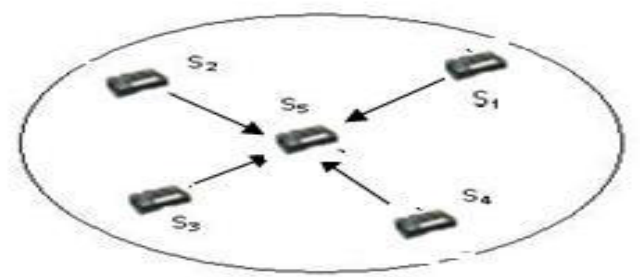

Fig.3. Cluster in DSN

1. Choose an ordering of variables: $\mathrm{S}_{1} \ldots \ldots . \mathrm{S}_{\mathrm{N}}$

For $\mathrm{i}=1$ to $\mathrm{S}_{\mathrm{N}}$

Add $\mathrm{S}_{\mathrm{i}}$ to the Network

Select CH (Parent) from $\mathrm{S}_{\mathrm{i}}$------------- $\mathrm{S}_{\mathrm{i}-1}$ such that $\mathrm{P}\left(\mathrm{S}_{1}, \mathrm{~S}_{2} \ldots \mathrm{S}_{\mathrm{N}}\right)=\Pi^{\mathrm{n}}{ }_{\mathrm{i}=1} \mathrm{P}\left(\mathrm{S}_{\mathrm{i}} \mid\right.$ parents $\left.\left(\mathrm{S}_{\mathrm{i}}\right)\right)=\mathrm{P}\left(\mathrm{S}_{\mathrm{i}} \mid \mathrm{S}_{\mathrm{i}}\right.$ $\left.--\mathrm{S}_{\mathrm{i}-1}\right)$ 
2. Calculate the probability of $\mathrm{CH}_{\mathrm{i}}=\mathrm{P}\left(\mathrm{S}_{\mathrm{i}} \mid \mathrm{P}\left(\mathrm{S}_{\mathrm{i}}\right)\right)=$ $\mathrm{P}\left(\mathrm{S}_{1}, \mathrm{~S}_{2}, \mathrm{~S}_{3}, \mathrm{~S}_{4}, \mathrm{~S}_{5}\right)=\mathrm{P}\left(\mathrm{S}_{5} \mid \mathrm{S}_{1}, \mathrm{~S}_{2}, \mathrm{~S}_{3}, \mathrm{~S}_{4}\right) \mathrm{P}\left(\mathrm{S}_{1}\right) \mathrm{P}$ $\left(\mathrm{S}_{2}\right) \mathrm{P}\left(\mathrm{S}_{3}\right) \mathrm{P}\left(\mathrm{S}_{4}\right)$

For i to $R_{N} \quad$ // for each round

If (The probability of $\mathrm{CH}_{\mathrm{i}}<=\mathrm{P}\left(\mathrm{S}_{\mathrm{i}}\right)$ )

\{ Else

Select next highest probability of node;

Selected as a cluster member; \}

3. Cluster Communication

If $\left(\mathrm{P}\left(\mathrm{S}_{\mathrm{N}}\right)>=\mathrm{T}_{\mathrm{H}} \mathrm{P}\right)$

Communication takes place between member nodes and $\mathrm{CH}$ in the network;

Else

Send to sleep mode in the network; \}

The selection of CHs in each cluster over the network, cluster member nodes start communication with $\mathrm{CH}$ as shown in Fig.3. Cluster members communicate with $\mathrm{CH}$ is based upon the node energy, bandwidth and link efficiency. The node working as $\mathrm{CHs}$ consumes more energy as compare to cluster member nodes for transmission of data to sink node. For each round, to select $\mathrm{CH}$ over the network, this is based on the highest probability among nodes in the cluster otherwise selected as cluster member. As the probability of each node decreases, then decreases in energy and bandwidth of each node in the network.

\section{H. Tabu Search Algorithm}

Tabu List: The initial solution becomes current solution and this solution is inserted into a memory list. The Tabu list is one of the mechanisms to prevent cycling and guide the search toward unexplored regions of the solution space. Tabu list is constructed by those recently visited solutions. It considered that size of the Tabu list depends upon the number of CHs.

Candidate List: TS make use of a candidate list that provides a list of moves to evaluate. Initially candidate list is assigned to NULL value.

Aspiration Condition: Aspiration condition will ensure the search process can release specific solution and enable efficient global optimization search when all candidate solutions are Tabu or a candidate solution better than the current optimal solution.

Nomenclature: $\{$ TabuList $\leftarrow$ null, candidateList $\leftarrow$ Null $\}$

An initial solution must be randomly generated to be chosen as the current solution among the $\mathrm{CH}_{\mathrm{i}}$, number of neighbor solutions of the current solution will be searched, finally the best of them will be chosen as the new current solution. During the TS, in order to prevent from the repeated search of the local optimal solution, reach the global optimal solution of the problem. As a result, TS uses a Tabu list to record the local optimal solutions that have been searched recently. Finally, return the best solution, which is solution of the optimization routing between $\mathrm{CH}_{\mathrm{i}}$ and sink node [29]. The TS algorithm is given as follows:

Step1. Generate an initial solution as randomly between the $\mathrm{CH}_{\mathrm{i}}$ and Sink node

Step2. Assign it as Best solution

While (not aspiration Condition ( )) // Searching for an optimal solution until aspiration condition is met for $\left({ }_{\mathrm{s}}\right.$ Candidate _ Neighbours $=$ True $)$

if (not containsTabuElements(sCandidate, tabuList)) // the neighboring solutions are checked

candidateList $\leftarrow$ candidateList + sCandidate end

end

sCandidate $\leftarrow$ LocateBestCandidate (candidateList)

if (fitness(sCandidate) $>$ fitness(sBest))

sBest $\leftarrow$ sCandidate

tabuList $\leftarrow$ featureDifferences(sCandidate, sBest)

while ( $\operatorname{size}($ tabuList $)>\operatorname{maxTabuListSize})$ ExpireFeatures(tabuList)

$$
\text { end }
$$$$
\text { end }
$$

end

return (sBest) //the final best solution

\section{A. Aspiration Condition $\left(\mathrm{CH}_{i}\right)$}

\{

Float $\mathrm{E}=$ Energy $\left(\mathrm{CH}_{\mathrm{i}}\right)$;

Float $\mathrm{B}=$ Bandwidth $\left(\mathrm{CH}_{\mathrm{i}}\right)$;

For (int $\mathrm{i}=1 ; \mathrm{i}<=n ; \mathrm{i}++$ )

\{

int $\mathrm{CH}_{\mathrm{i}}=\mathrm{i}$;

Switch $\left(\mathrm{CH}_{\mathbf{i}}\right)$

\{

Case 1: if $(\mathrm{E}=$ Max \& \& $\mathrm{B}=$ High $)$

Return best Aspiration Condition;

Break;

Case 2: if $(\mathrm{E}=$ Fair \&\& $\mathrm{B}=$ Fair $)$

Return Average Aspiration Condition;

Break;

Case 3: if $(\mathrm{E}=$ Min \&\& $\mathrm{B}=$ Min $)$

Return worst Aspiration Condition;

Break;

Default: case 4: if ( $\mathrm{E}<=$ Threshold Level energy \&\& $\mathrm{B}<=$ Threshold_level_ Bandwidth)

Return false;

\}


Let ' $\mathrm{CH}_{\mathrm{i}, \mathrm{j}}$ ' be the total distance from cluster head node to sink node. The fitness function of the neighboring solution can be computed by using Eq. (13).

Fitness $\left(\mathrm{CH}_{\mathrm{i}, \mathrm{j}}\right)=\sum_{n=1}^{n} 1 / \mathrm{C}_{\mathrm{CHi}} / /$ the total distance from cluster head node ${ }_{\mathrm{i}}$ to sink node

Where,

$\mathrm{C}_{\mathrm{CHi}}=$ be the distances between the cluster heads.

\section{SIMULATION}

To simulated the proposed scheme for various network scenarios using ' $\mathrm{C}$ ' programming language with a confidence interval of $95 \%$. We compare results of proposed work with an existing $\mathrm{LEACH}$ protocol with $\mathrm{CH}$ selection and optimization of routing for DSNs. This section presents the simulation model, simulation procedure, and results and discussions.

\section{A. Simulation Model}

Network model: Considered an area of $1 * b$ square meters for DSN. A network consists of $\mathrm{S}_{\mathrm{N}}$ static nodes that are randomly deployed within a given area. The bandwidth is $\mathrm{BW}_{\text {single-hop }}$ for a single hop connected sensor nodes.

Channel model: S-MAC protocol [30] is used for media access. The transmission of packets is assumed to occur in discrete time at nodes as well $\mathrm{CHs}$. We have considered the channel to be error free and contention between sensor nodes.

Propagation model: A free space propagation model is used with propagation constant ' $\beta$ '. Transmission range of DSN node (communication radius) is $\mathrm{R}$ for a single hop distance. It is assumed that at any given time, the value of transmitted energy per packet is ' $E$ ' Joules for every node. $R$ is directly proportional to e of the node. That is, $\mathrm{R}=\mathrm{CE}$, where constant of proportionality ' $C$ ' depends on the medium of communication, attributed to ' $\beta$ '.

To measure the performance parameters such as searching time, search success rate, node energy consumption (mJoules), network lifetime, and time efficiency of optimization of routing.

\section{B. Simulation Procedure}

Simulation inputs for proposed scheme are as follows: Number of Nodes $\left(\mathrm{S}_{\mathrm{N}}\right)=500$, Energy of each nodes $\left(\mathrm{E}_{\mathrm{N}}\right)=$ 2Joules, Number of sink nodes $(\mathrm{Ns})=1$, Size of the network $=$ $5000 * 5000$ meters, Transmission range $\left(T_{R}\right)=100$ meters, Energy required for sensing of each node $\left(E_{S}\right)=50 \mathrm{~nJ} / \mathrm{Bit}$, Energy required for transmission of data $\left(E_{T}\right)=50 \mathrm{~nJ} / \mathrm{Bit}$, Size of packets $(\mathrm{P})=64 \mathrm{Bits}, 128 \mathrm{Bits}$, 512Bits, 1024Bits and so on, and Threshold Level Energy $\left(\mathrm{T}_{\mathrm{H}} \mathrm{E}\right)=0.05$ Joules, Transmission of data $=$ bits $/ \mathrm{sec}$ and bandwidth of the network $\left(\mathrm{B}_{\mathrm{N}}\right)=4 \mathrm{mbps}$.
Begin

1) Deploy the number of nodes randomly as in DSN environment.

2) Construct the number of cluster in the related area

3) To select cluster head in their specified clusters

4) Apply the proposed scheme DSN.

5) Compute the performance parameters.

End

6) Generate graphs.

\section{Performance Parameters}

The following performance parameters are assessed:

1) Search Time for $\mathrm{CH}$ Selection: It is defined as the number of nodes increases as the increment of search time in DSN. It is expresses in terms of milliseconds (msecs).

2) Search Success rate for Optimization of Routing: It is defined as the number of transmitting nodes ( $\mathrm{CHs})$ increases as the increases the search success rate in an environment. It is expressed in terms of percentage (\%).

3) Energy Consumption for Optimization of Routing: It is defined as the total energy consumed for the optimization of routing in DSN. It is expressed in terms of 'mJoules'.

4) Time Efficiency for Optimization of Routing: It is defined as the time required for optimization of routing with given number of transmitting CHs. It is expressed in terms of percentage $(\%)$.

5) Network Lifetime: As the number of rounds increases, the network lifetime decreases in DSN. It is expressed in terms of percentage $(\%)$.

6) Packet Delivery Ratio (PDR): It is defined as the number of rounds increases, the PDR decreases in DSN with given number of $\mathrm{CHs}$ increases. It is expressed in terms of percentage $(\%)$.

\section{Results and Discussions}

Fig. 4 presents searching time for $\mathrm{CH}$ selection with given number of nodes in DSNs. As the number of nodes increases, searching time for selection of $\mathrm{CHs}$ is increases in DSN. The proposed $\mathrm{BN}$ approach is more efficient than the $\mathrm{LEACH}$ protocol because $\mathrm{CH}$ selection is based on the highest probability of among nodes in the cluster. But LEACH protocol is used to select the $\mathrm{CHs}$ based on randomly in the network, which result in less optimal selection of $\mathrm{CHs}$ and delay will be more.

Fig. 5 explains the search success rate for optimization of routing over the $\mathrm{CHs}$ in DSN environment. As the number of transmitting nodes or $\mathrm{CHs}$ increases, searching rate of the optimization of routing decreases. The proposed TS algorithm is more efficient than the LEACH algorithm with given number of transmitting nodes. In LEACH protocol end to end delay and dead nodes are more because of randomized rotation of CHs. It will work on the basis of two phase schemes are: setup phase and steady - state phase. In this work, achieved search rate of optimization of routing is $95 \%$ for $25 \mathrm{CHs}$, and less delay will be considered in the proposed work. 


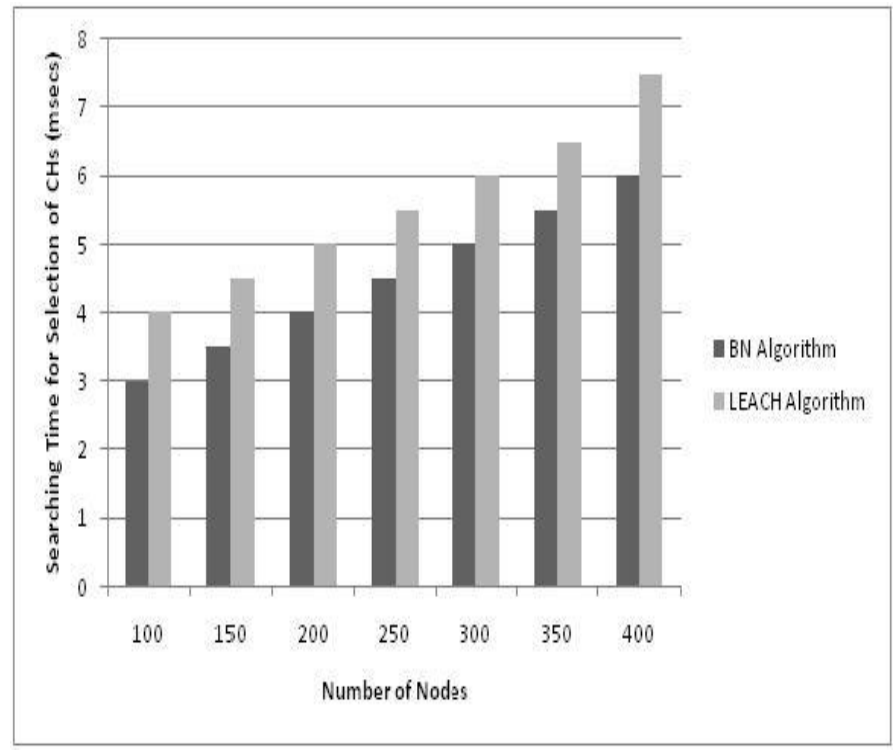

Fig. 4. Searching Time for Selection of CHs Vs Number of Nodes

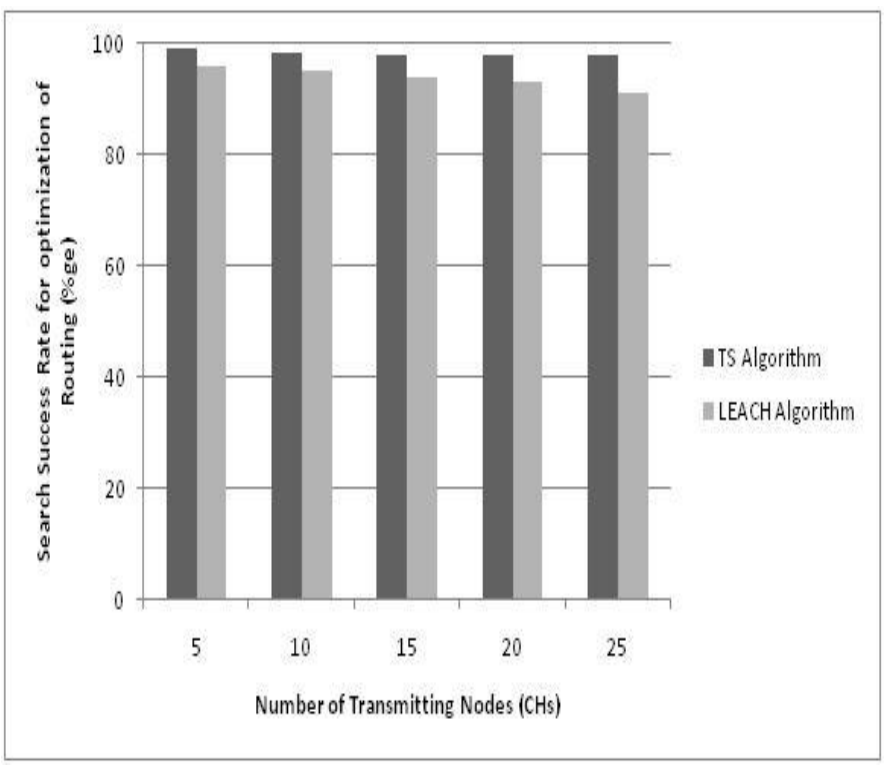

Fig.5. Search Success Rate for Optimization of Routing Vs Number of Transmitting Nodes

Fig.6 describes the energy consumption for optimization of routing with number of $\mathrm{CHs}$ in the DSN environment. With increase the number of $\mathrm{CHs}$, the energy consumption increases in both proposed algorithm (CBTS protocol) and LEACH protocol. The proposed protocol performs better compared to LEACH. The energy consumption is due to optimization of routing in distributed environment. The proposed CBTS protocol exhibits less energy consumption because its uses neighboring solutions for path computations. The LEACH protocol requires more energy consumption because of its more end to end delay for path computations.
Fig.7 presents time efficiency for optimization of routing with number of CHs. As the number of transmitting nodes (CHs) increases, time efficiency of optimization of routing decreases in DSN environment. As the proposed CBTS uses only neighboring solutions which are based on the Tabu list, aspiration condition, candidate list and fitness function. The time efficiency involved will be less unlike the LEACH algorithm do not uses any heuristic functions.

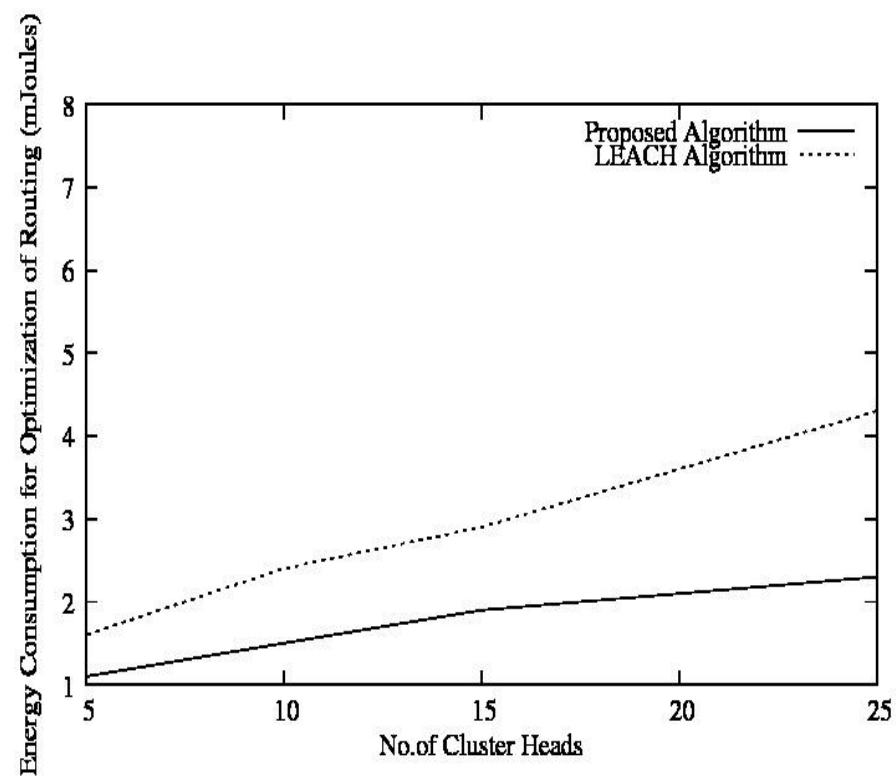

Fig. 6. Energy Consumption for Optimization of Routing Vs Number of Cluster Heads

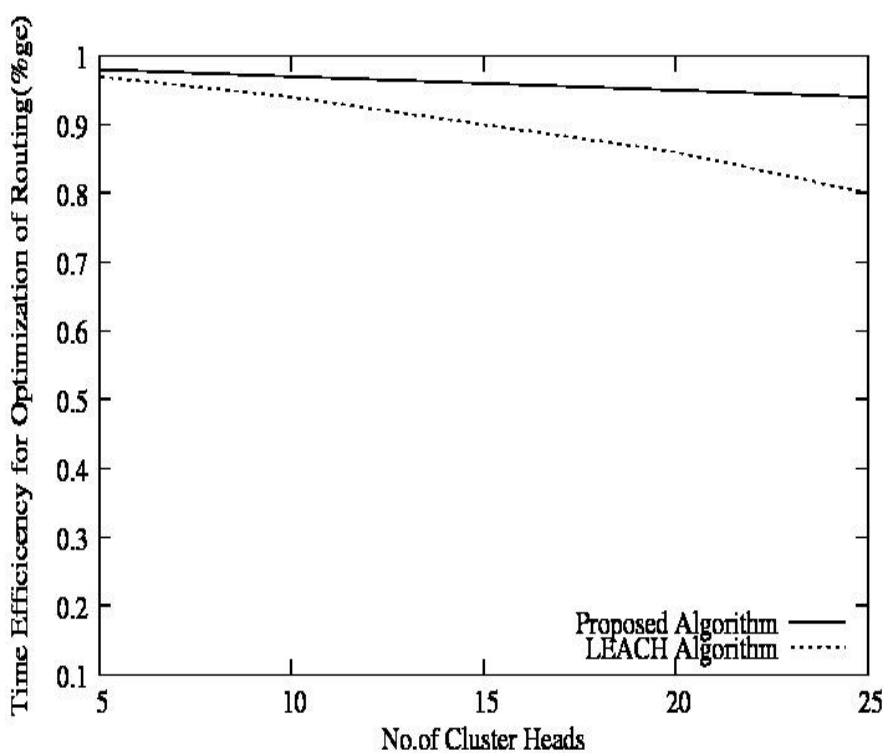

Fig.7. Time Efficiency for Optimization of Routing Vs Number of Cluster Heads 


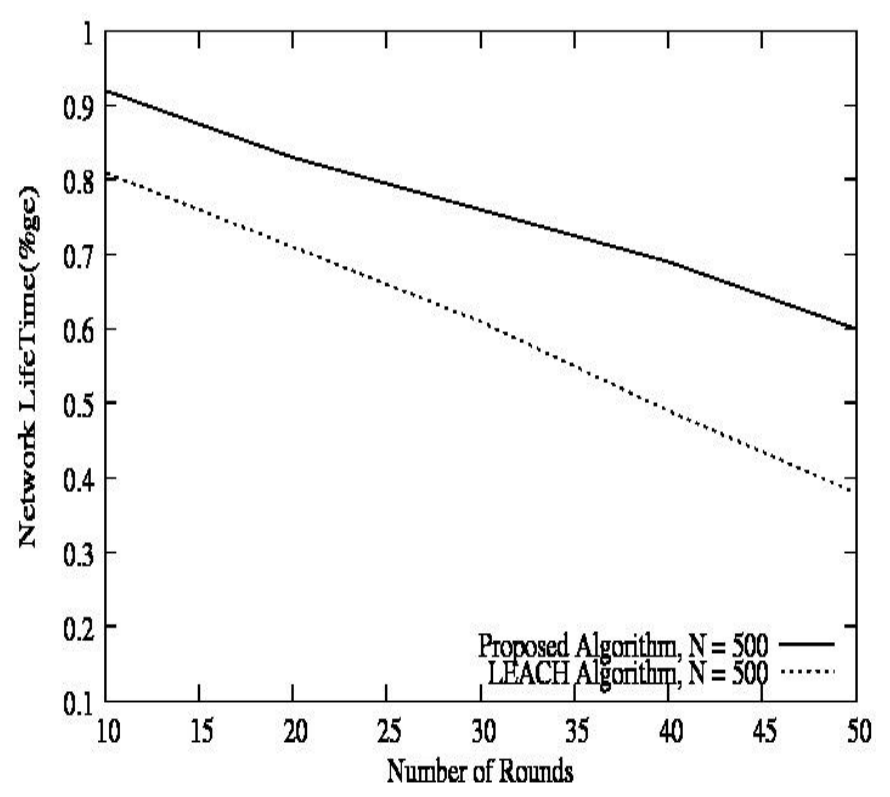

Fig. 8. Network Lifetime Vs Number of Rounds

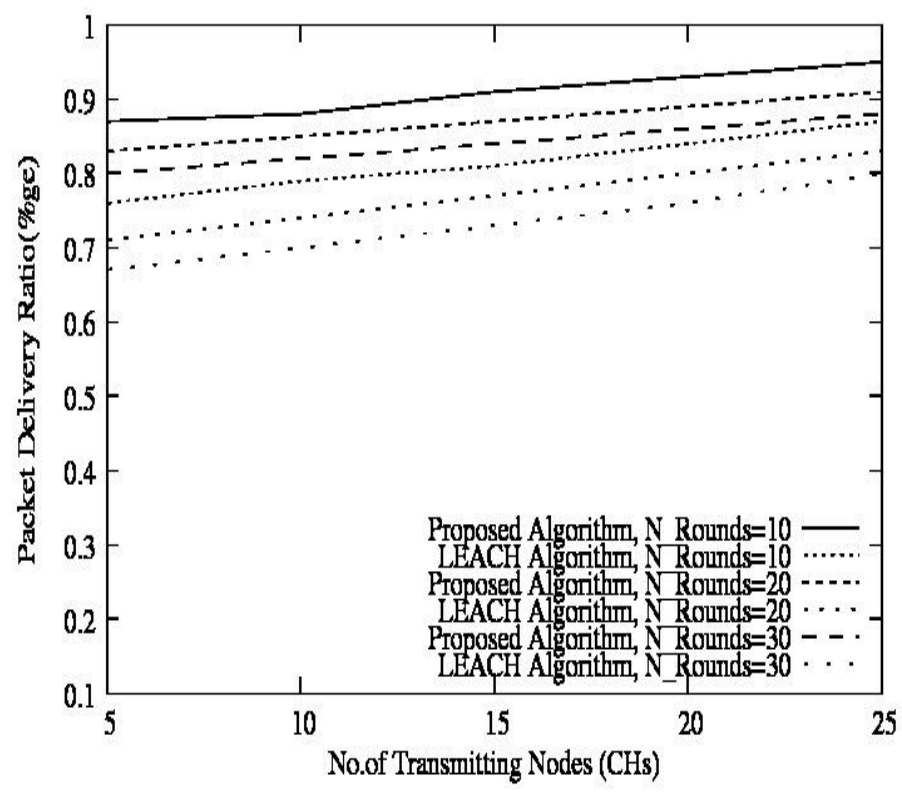

Fig. 9. Packet Delivery Ratio Vs Number of Transmitting Nodes

Fig.8 explains the network lifetime of network with given number of rounds. With increase the number of rounds along with given number nodes $(\mathrm{N}=500)$, the network lifetime decreases in proposed algorithm and LEACH algorithm. After 50 rounds is achieved to $60 \%$ of network lifetime of the network because of its less dead nodes and energy consumption of each node as compared to LEACH algorithm. If the energy of a generic node is under the threshold level $(0.05 \mathrm{~J})$, then that node is sent to sleep mode in the network for fault recovery.

Fig. 9 describes the packet delivery ratio with number of transmitting nodes $(\mathrm{CHs})$. As the number of $\mathrm{CHs}$ increase the increases in the packet delivery ratio of the network. In this work, with increase in the number of rounds, the packet delivery ratio decreases. The proposed algorithm uses more packet delivery ratio as compared to LAECH protocol. The LEACH algorithm will considered as less packet delivery ratio because of its head changes and its advertisements of information with neighboring nodes, it's more energy consumption of node and dead nodes.

\section{CONCLUSION}

The proposed method is energy efficient and responsive to network. It includes $\mathrm{CH}$ selection and optimization of routing. The proposed system selects efficient $\mathrm{CHs}$, optimization of routing and increase the network lifetime. It has been observed that $\mathrm{BN}$ is used to probabilistically select $\mathrm{CHs}$ in achieving better results as compare with LEACH approach. TS method is used for optimization of routing in DSN. It has been observed that the performance of TS is robust and fast. The TS reaches the optimal solution faster. As compared to LEACH protocol the proposed model performed better performance in the network. A simulation results obtained from a proposed algorithms are more efficient than LEACH algorithm.

\section{REFERENCES}

[1] Iyengar. S., S, Ankit. T, Brooks. R., R, An Overview of Distributed Sensors Network.

Available:

http://books.google.com/books/about/Distributed-sensor-networks.html ?id= Nff5

[2] Shivakumar. S, Iyengar. S., S, Taxonomy of Distributed Sensors Network.

Available:

http://systems.ihpmicroelectronics.com/uploads/downloads/DS1 2007 06_Taxonomy.pdf

[3] Heinzelman. W., C, Balakrishnan. H, Energy-efficient Communication Protocol for Wireless Micro Sensor Networks. In the Proceedings of the $33^{\text {rd }}$ Annual Hawaii International Conference on System Sciences, pp. 1-10, 2000.

[4] Karaki. A., N, Kamal, A., E, Routing Techniques in Wireless Sensor Networks: A Survey, Journal on IEEE wireless Communications, vol 11, pp. 6-28, 2004

[5] Allirani. A, Suganthi. M, An Energy Efficient Cluster Formation Protocol with Low Latency in Wireless Sensor Networks, International Journal of Electrical and Computer Engineering, vol.4, no. 16, pp. $985-$ 991, 2009.

[6] Handy. M., J, Marc. H, Dirk. T, Low Energy Adaptive Clustering Hierarchy with Deterministic Cluster-Head Selection, In proceedings of 4th International workshop on mobile and wireless communications network, pp. 368 - 372, 2002.

[7] Yun. Li., N, Weiyi. Z, Weiliang. Z, Xiaohu.Y, Mahmoud. D, Enhancing the Performance of LEACH Protocol in Wireless Sensor Networks, In proceedings of IEEE conference on computer communications, pp. 223 $-228,2011$

[8] Michel. G, Jean-Yves. P, Tabu Search. pp. 165-188. Available:

http://www.inf.ufpr.br/aurora/disciplinas/topicosia2/livros/search/TS.pdf

[9] Anant. O, Wichai. S, Boonruang. M, Thanatchai. K, Tabu Search Approach to Solve Routing Issues in Communication Networks. In Proceedings of World Academy of Science: Engineering \& Technology, vol. 53, pp. 1168-1171, 2009

[10] Bager. Z, Mohammad. Z, Vahid. M., N, A Novel Cluster Based Routing Protocol in Wireless Sensor Networks, International Journal of Computer Science Issues, vol. 7, no 1, pp. 32 -36, 2010.

[11] Young.g., H, Heemin. K, Yung. C.,. B, Energy Efficient Fire Monitoring Over Cluster Based Wireless Sensor Networks, International Journal of Distributed Sensor Networks, vol. 2012,pp. 1-11, 2012. 
[12] Ali. N, Faezeh. S., B, Abdul. H., Z, A New Clustering Protocol for Wireless Sensor Networks using Genetic Algorithm approach, Journal of Wireless Sensor Network, vol.3, no. 11, pp. 362-370, 2011.

[13] Sanghak. L, Junejae. Y., C, Distance based Energy Efficient Clustering for Wireless Ssensor Networks. In proceedings of $29^{\text {th }}$ Annual IEEE international Conference on Local Computer Networks, pp. 567 - 568, 2004.

[14] Taewook. K, Jangkyu. Y, Hoseung L, Icksoo. L, Hyunsook. K, Byunghwa. L, Byeongiik. L, K, A Clustering Method for Energy Efficient Routing in Wireless Sensor Networks, In Proceedings of the $6^{\text {th }}$ WSEAS International Conference on Electronics, Hardware, Wireless and Optical Communications, pp. 133 - 138, 2007.

[15] Jyh. H., C, Rong. H., J, An Energy-Aware, Cluster-Based Routing Algorithm for Wireless Sensor Networks, Journal of Embedded and Ubiquitous Computing, vol. 3824, pp. 255-266, 2005.

[16] Arroyo. V., R, Marques. A., G, Vinagre-Diaz. J, Cid-Sueiro. J, A Bayesian Decision Model for Intelligent Routing in Sensor Networks, In proceedings of $3^{\text {rd }}$ International Symposium on Wireless Communication Systems, pp. 103 - 107. 2006

[17] Bhaskar. K, Sitharama. I, Distributed Bayesian Algorithms for FaultTolerant Event Region Detection in Wireless Sensor Networks, IEEE Transactions on computers, vol. 53, no. 3, pp. 241- 250, 2004.

[18] Mohammad. M, Subhash. C, Rami. A, Bayesian Fusion Algorithm for Inferring Trust in Wireless Sensor Networks, Journal of Networks, vol 5, no 7, pp. 815-822, 2010

[19] Abdelmorhit. R, Samuel. P, Tabu Search Algorithm for Cluster Building in Wireless Sensor Networks, IEEE Transactions on Mobile Computing, vol. 8, no. 4, 2009, pp. 433-444, 2009.

[20] Masaya. Y, Kazuo. O, Ant Colony Optimization Routing Algorithm with Tabu Search, In proceedings of International conference of engineers and computer scientist, vol. 3, pp. 1-4, 2010

[21] Nadjib. A, Nadjib. A, Khaled. B, Guy. P, Tabu Search WSN Deployment Method for Monitoring Geographically Irregular Distributed Events. Journal of sensors, vol.9, pp. 1625-1643, 2009.
[22] Thamilselvan. R, Balasubramanie. P, Integrating Genetic Algorithm, TS approach for job shop scheduling, International Journal of Computer Science and Information Security, vol. 2, no. 1, pp 1-6, 2009.

[23] Eneko. O, Fernando. D, Comparison of a Memetic Algorithm and a Tabu Search Algorithm for the Traveling Salesman Problem. In the Proceedings of the Federated Conference on Computer Science and Information Systems, pp. 131-136, 2012.

[24] Bajeh A. O. Abolarinwa K. O, Optimization: A Comparative Study of Genetic and Tabu Search Algorithms. International Journal of Computer Applications, vol. 31, no. 5, pp. 43-48, 2011.

[25] Ben.G, Rugari. F, Futtin. F, Kuretti. R, Bayesian Networks, Encyclopedia of studies in Quality and Reliability, Wiley and Sons, 2007.

[26] Nishant. S, Upinderpal. S, A Location Based Approach to Prevent Wormhole Attack in Wireless Sensor Networks. International Journal of Advanced Research in Computer Science and Software Engineering, vol.4, no.1, pp. 840-845, 2014.

[27] Herbert. T, Donald. L, Schilling principles of communication, systems. McGraw-Hill 1986.

[28] Miao. G, Himayat. N, Li. G., Y, Energy efficient link adaptation in frequency-selective channels. IEEE Transaction on Communication, vol. 58 , no.2, pp. 2010

[29] Lokesh B., B, Nalini. N, S.R.,Nagaraj, H.V., Chaitra, N. Srinivas, Performance Analysis of Heuristic Techniques for Optimization of Routing in Distributed Sensor Networks. In the proceedings of International Conference on Emerging Research in Computing, Information, Communication and Applications, pp. 547-556, 2013

[30] Heinzelman. W, Chandrakasan. A, Balakrishnan. H. Energy efficient communication protocol for wireless micro sensor networks. In the Proceedings of the IEEE Hawaii international conference on system sciences, vol.8, pp. 8020-8030, 2000 